\title{
Can VEGF reverse diabetic neuropathy in human subjects?
}

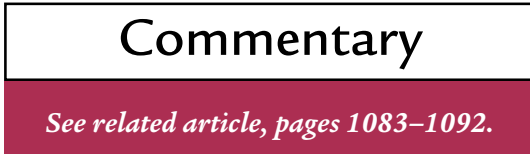

\author{
Aristidis Veves ${ }^{1}$ and George L. King ${ }^{2}$ \\ ${ }^{1}$ Microcirculation Lab, Beth Israel Deaconess Medical Center, and \\ ${ }^{2}$ Research Division, Joslin Diabetes Center, Harvard Medical School, Boston, Massachusetts, USA
}

Address correspondence to: George L. King, Joslin Diabetes Center, One Joslin Place, Harvard Medical School,

Boston, Massachusetts 02215, USA. Phone: (617) 732-2622; Fax: (617) 732-2637; E-mail: George.King@joslin.harvard.edu.

Peripheral polyneuropathy is a common complication of diabetes that can clinically affect $30 \%$ of all diabetic patients and is the most common form of diabetic neuropathy. The main symptoms are numbness, tingling, burning, and shooting pain in the legs, whereas the physical findings include reduced pain, touch, and vibratory perception in a symmetrical stocking glove distribution $(1,2)$. Loss of protective sensation allows unperceived trauma and pressures of daily ambulation to cause foot ulcers, which can lead to prolonged immobilization or even amputation of the lower limb.

The main histologic changes of diabetic polyneuropathy are loss of myelinated and unmyelinated fibers and segmental demyelination. Changes in the myelinated fibers can be measured functionally in vivo by quantitating nerve conduction velocities and amplitudes of evoked responses, which are objective techniques and can assess nerve function without relying solely on the patient's subjective responses. In contrast, testing of small fibers is more difficult, as it is subjective and dependent on the patients' active participation (3).

Hyperglycemia, which has emerged as a major risk factor for the development of diabetic neuropathy, may affect the peripheral sensory nerves through several mechanisms (2). Several of the current hypotheses are shown in Figure 1. First, the increased flux through the polyol pathway in hyperglycemic patients may lead to intracellular sorbitol accumulation and, potentially, to osmotic increase or changes in the $\mathrm{NAD} / \mathrm{NADH}$ ratio induced by the flux through the aldose reductase pathway. These changes can cause direct neuronal damage or decrease neuronal blood flow, indirectly leading to peripheral nerve hypoxia. Second, the activation of protein kinase $\mathrm{C}(\mathrm{PKC})$ in response to increased diacylglycerol lev- els via the de novo synthesis pathway can affect the Na, K ATPase, and other enzymes that are important for maintaining cellular membrane potential and nerve conduction. In addition, PKC activation can induce vasoconstriction and reduce neuronal blood flow. Third, the auto-oxidation of glucose causes increased production of reactive oxygen species and the formation of advanced glycation end products (AGEs) by nonenzymatic glycation of proteins. AGEs then bind to a cell-surface receptor and cause activation of the NF- $\mathrm{KB}$, which is associated with endothelial dysfunction and reduced nerve blood flow. Finally, diabetes impairs the hepatic $\Delta-6$ desaturation of dietary linolenic acid to $\gamma$-linolenic acid and results in reduced synthesis of vasoactive prostanoid in the vasa nervorum. This defect leads to reduced endoneurial blood flow and nerve hypoxia. Each of these models finds some support in the various biochemical abnormalities seen in peripheral nerves and vasculature of diabetic patients.

Other physiological changes that accompany the onset of diabetes may also contribute to peripheral neuropathy. In particular, decreased blood flow to these nerves is one of the earliest functional findings in the development or induction of diabetes. The resulting local hypoxia in the peripheral nerves is believed to be a major pathogenic factor, although impaired mitochondrial functions and apoptosis of neurons and Schwann cells also occurs with similar timing and may act independently of hypoxia to induce peripheral nerve dysfunction. In addition, reduction in neurotrophic factors such as nerve growth factor availability (including neurotrophin-3 [NT-3], brainderived neurotrophic factor, and neurotrophin-4/5 [NT-4/5]) and aberrant phosphorylation of the neurofilaments that are responsible for the structural nerve axon integrity have also been implicated in the pathogenesis of diabetic neuropathy.

\section{Microvascular changes in diabetes}

In 1959, Fagerberg first described the thickening and hyalinization of the walls of the nerve vessels and suggested that these changes might explain the development of diabetic neuropathy (4). Subsequent studies have confirmed the presence of endoneurial microangiopathy characterized by basement membrane thickening, endothelial cell hyperplasia and hypertrophy, and pericyte cell degeneration. These microvascular changes are not specific to the peripheral nerves, but occur in all organs and tissues in the diabetic state. Although these findings suggest that blood flow to the nerve is decreased, the lack of reliable, noninvasive techniques that can directly measure nerve blood flow has hampered the collection of data that can confirm or refute this hypothesis. The clearest supportive evidence is the demonstration of reduced sural endoneurial oxygen tension and epineurial oxygen saturation in the rat diabetes role (5). On the other hand, a recent study employing laser Doppler flowmetry failed to show any association between sural nerve blood flow and early peripheral neuropathy in diabetic patients (6). Therefore, in contrast to the experimental neuropathy discussed below, the magnitude of nerve flow impairment is not clear for clinical diabetic neuropathy. As a consequence, nerve blood flow cannot be employed as an efficacy endpoint in clinical trials.

Good glycemic control, the only proven treatment for diabetic neuropathy in humans (1), is only known to prevent the development or halt the progression of the disease and has not been shown to reverse established lesions. A number of other interventions have been tested, including treatment with aldose 


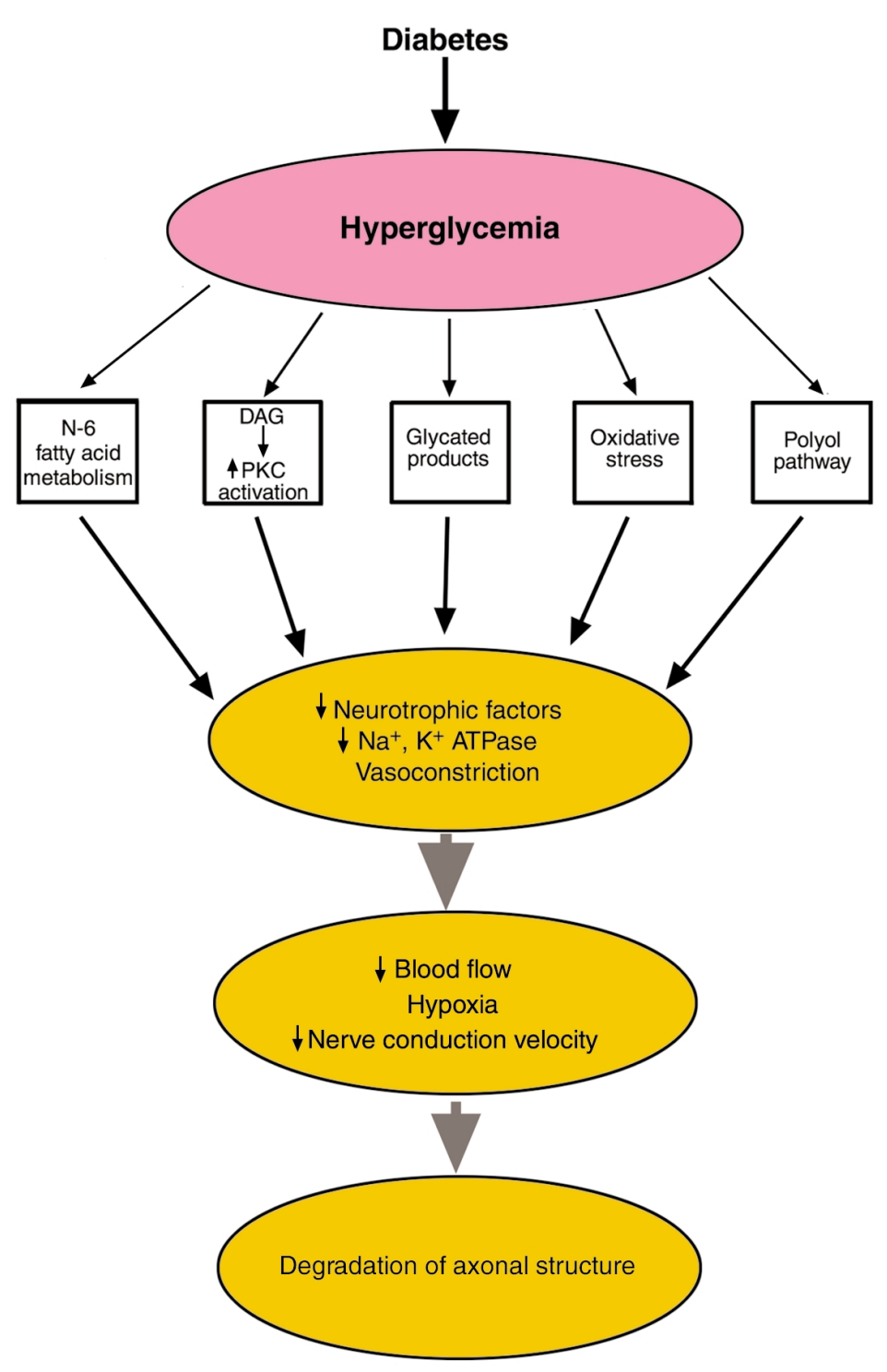

Figure 1

Pathogenesis of diabetic neuropathy. Factors implicated in the pathogenesis of diabetic neuropathy include the activation of the polyol pathway, the activation of protein kinase $C(P K C)$, increased oxidative stress, the impaired $\mathrm{N}-6$ fatty acid metabolism, auto-oxidation of glucose, the formation of advanced glycation end products (AGEs), and the reduced bioavailability of neurotrophic factors. All these mechanisms are interrelated and can potentiate each other's detrimental effects. Although the exact mechanisms of their action are not well understood, it is currently believed that these factors lead to reduced $\mathrm{Na}^{+}, \mathrm{K}^{+}$ATPase activity and vasoconstriction, reduced endoneurial blood flow and nerve hypoxia. The latter changes then lead to reduced nerve conduction velocities, axonal loss, axonal demyelination, and nerve dysfunction. DAG, diacylglycerol.

reductase inhibitors, $\gamma$-linolenic acid, antioxidants such as $\alpha$ lipoic acid, and nerve growth factors (7). Despite initial encouraging reports with most of these agents, the results of large perspective controlled clinical trials have invariably been negative, and none of the tested medications have been approved for use in this country. Therefore, an urgent need exists to develop new therapeutic approaches that will improve nerve function in diabetic patients. physiology. Changes in the unmyelinated fibers can be tested only indirectly by employing behavioral tests, such as the tailflick response to a thermal noxious stimulus.

In contrast to human neuropathy, ample data are available in experimental neuropathy regarding the changes in nerve blood flow. Cameron et al. (9), using microelectrode polarography and hydrogen clearance measurements, have measured a $40 \%$ reduction in endoneurial blood flow in streptozotocin-induced diabetic rats, beginning within a week of inducing diabetes, and persisting over a 4-month observation period. Laser Doppler flowmetry measurements in the sciatic nerve of streptozotocin-induced diabetic rats and spontaneously diabetic $\mathrm{BB}$ rats have yielded similar results (10). Nerve blood flow is therefore routinely used as an end point in assessing new possible therapeutic agents in diabetic animals.

\section{VEGF and diabetes}

VEGF is a potent selective mitogenic cytokine for endothelial cells, and its expression can be induced by hypoxia through the hypoxia-inducible factor-1 (HIF-1) (11). VEGF binds to several receptors, of which VEFG receptor 1 and 2 are the best characterized. Under normal conditions, the receptors are expressed at low levels, but, as is seen with VEGF itself, tissue hypoxia potently stimulates overexpression of these receptors. Hypoxia-induced VEGF and VEGF receptor expression promotes endothelial cell proliferation and migration, leading to angiogenesis and the development of collateral neovascularization in ischemic tissues (12).

Because systemic factors such as diabetes, hypercholesterolemia, and aging are reported to impair VEGF expression in the heart and lower extremities, VEGF, administered either through gene therapy or protein injection, has been proposed to enhance collateral vessel growth and prevent tissue necrosis under ischemic conditions (13). This approach to therapeutic angiogenesis is currently under intensive investigation and can be of particular benefit to diabetic patients with coronary or peripheral vascular disease. However, VEGF overexpression can also have a detrimental effect in the retina (14). More specifically, retinal hypoxia in the diabetic state due to reduction of blood flow stimulates the expression of VEGF and results in retinal neovascular- 
ization and increased retinal vascular permeability. Macular edema, bleeding, fibrosis, and loss of vision may follow. Local and systemic VEGF antagonists have been proposed as potential therapeutic interventions for the treatment of diabetic macular edema and proliferative retinopathy (15). Conversely, treatment with exogenous VEGF may well exacerbate these disorders. Another adverse effect of VEGF is that it significantly augments vascular permeability, and its use in clinical trials has been associated with the development of peripheral edema. Finally, it is also of some concern that in vitro studies have shown that VEGF activates the PKC pathway, which may contribute both to diabetic retinopathy and to neuropathy (Figure 1; see also ref. 16).

\section{VEGF and diabetic neuropathy}

Very little information is available regarding the role of VEGF in the development of diabetic neuropathy. While the peripheral nerves of diabetic patients are clearly hypoxic, it is not certain whether this hypoxia can lead to increased VEGF expression, as occurs in muscle and the retina. In streptozotocin-induced diabetic rats, VEGF expression has been reported to be increased in the sciatic nerve and dorsal root ganglia. Treatment with insulin and/or nerve growth factor can prevent the increases in VEGF expression (17).

VEGF gene transfer was initially tested on experimental ischemic peripheral neuropathy by Isner and his colleagues (18). In a recent study, this group reported that transfer of naked DNA encoding VEGF into ischemic muscle of rabbits could slow or reverse the development of reduced nerve conduction velocities and sensory nerve action potentials. Furthermore, VEGF gene therapy was shown to prevent or reverse the establishment of axonal loss and myelin degeneration that was observed in the untreated animals with similar degrees of hindlimb ischemia. Blood flow at the nerve level, measured by Laser Doppler perfusion imaging and fluorescent BS-1 lectin staining, was also found to be preserved at normal levels in the VEGF-treated animals where it was considerably reduced in the untreated ischemic animals. Finally, it was also reported that VEGF stimulated the migration and prevented the hypoxia-induced apoptosis of Schwann cells in vitro, which exhibited VEGF receptors. Therefore, the authors sug- gested that VEGF, in addition to restoring blood flow by inducing angiogenesis, may directly promote the survival of peripheral nerve cells. Such characteristics would make VEGF an ideal agent for preventing or restoring nerve dysfunction in diabetes.

In a recent issue of the JCI, the same group of investigators advanced their observations by reporting the effect of VEGF gene transfer on experimental diabetic neuropathy (19). Using a design similar to that in the previous report, they have studied peripheral nerve functions in streptozotocin-induced diabetic rats. Large nerve fibers were tested by measuring nerve conduction velocities, while the small fiber function was assessed by testing the tailflick in response to thermal noxious stimulus. Remarkably, VEGF gene transfer 12 weeks after the induction of diabetes fully restored nerve function abnormalities studied in both large and small fibers. Furthermore, VEGF restored nerve blood flow and nerve vessel numbers to levels that were observed in nondiabetic animals, indicating that VEGF exerts its beneficial effects by promoting angiogenesis in the peripheral nerves. The authors observed similar effects in alloxan-induced diabetic rats, indicating that these effects are not specific to a single animal model. Although it is tempting to speculate that considerable normalization in nerve pathology must underlie the observed improvement in nerve electrophysiology, this hypothesis remains to be tested by histological analysis.

\section{Questions and concerns}

As with all original, well-conducted studies, the present one creates as many questions as it answers. The first question to be addressed is how representative are the chosen animal models to human diabetic neuropathy. Both rats and rabbits in this, and the vast majority of other studies, have severely uncontrolled diabetes that makes them prone to dehydration and can induce a severe catabolic state. In this study, this can be best observed by the reduced weight of the diabetic rats when compared with the nondiabetic animals. Therefore, VEGF may act by reversing the catabolic stage locally, through the increase in the blood flow in the affected limb, rather than by reversing the conditions that are specifically related to the development of diabetic neuropathy in humans. The fact that VEGF induced similar effects in the ischemic rabbit hindlimb model further supports this possibility, as lower limb ischemia in humans does not cause neuropathy severe enough to be comparable to that observed in diabetes. Finally, it is also of interest that VEGF expression declined in untreated diabetic animals in the present study, in contrast to a previous report that indicated VEGF expression increases in untreated diabetes (17). This difference in the previous study by Samii et al. and the present study could be due to the different metabolic state of the animals.

Another basis for healthy skepticism can be derived from the fact that VEGF is only one of many factors to be shown to have these dramatic effects on experimental neuropathy in rodents. A number of studies have shown similar reversal of nerve function and blood flow to normal levels by a variety of factors in experimental neuropathy only to fail to affect human neuropathy. Such factors include aldose reductase inhibitors, vasodilators such as prazosin, nifedipine, angiotensin-converting enzyme inhibitors, ET-1 antagonists, aminoguanidine that inhibits the formation of AGEs, $\gamma$-linolenic acid, nerve growth factors, acetyl-L-carnitine, and desforoxamine (20). This has prompted investigators to question the validity of the experimental rodent models as representative of human diabetic neuropathy.

\section{Clinical outlook}

As shown in Figure 1, human neuropathy is the result of multiple factors, so it may be too optimistic to believe that reversing one of them will halt or reverse nerve damage. The notion of targeting multiple mechanisms simultaneously by administering combination treatments is therefore winning converts among clinical investigators. Possible combinations include antioxidants, aldose reductase inhibitors, nerve growth factors, and/or PKC inhibitors $(2,5,7,21)$. However, before such studies are conducted, detailed information will be required about each of the factors that contribute to the disease. Despite legitimate reservations about the applicability to human patients of findings in experimental diabetes, well-controlled studies provide essential guidance for clinical treatment of diabetic neuropathy.

The possible use of VEGF, which can have a direct impact on both the nerve 
blood flow and the nerve cells, offers distinct advantages over other therapeutic approaches that target either of these tissues separately. The fact that intramuscular VEGF gene transfer is a simple method that does not require elaborate techniques makes it a good candidate for clinical use in the future. Because the lower limb is often ischemic in diabetes, intramuscular VEGF transfer may help control not only nerve ischemia, but muscle ischemia as well.

Cautious attention should be paid, however, to the possible adverse effects of VEGF, particularly the development of proliferative retinopathy, a condition that is not present in the diabetic rat. Limited experience so far indicates that intramuscular VEGF gene transfer does not exacerbate retinopathy in humans, but considerably more data will be required before firm conclusions are justified. Furthermore, VEGF can cause peripheral edema in the lower extremity, and this can have grave consequences in the diabetic neuropathic patient. More specifically, the neuropathy-related insensitivity may not allow patients to feel that the increased size of their feet makes their shoes too small, causing the shoes to apply enough pressure on the skin to disrupt blood flow and cause skin necrosis. The possible mitogenic effects of VEGF in tumor development should also be kept in mind.

VEGF gene therapy is currently being tested in treating lower limb ischemia and promoting wound healing in diabetic and nondiabetic patients. The results of Schratzberger et al. (19) suggest that the effect of VEGF on nerve function should be included in the endpoints of such trials. Such preliminary data can be collected by using simple, noninvasive, easily performed methods and will not interfere with the original design of the study. Further clinical trials should be contemplated if preliminary data are encouraging.

\section{Acknowledgments}

The authors wish to express their appreciation to Ronald J. Burke, Jr. for his administrative assistance in the preparation of this manuscript. The work in this article is supported by NIH grants R01 EY 05110 and EY 09178, and NIDDK grant 3683659725 .

1. Vinik, A.I., Park, T.S., Stansberry, K.B., and Pittenger, G.L. 2000. Diabetic neuropathies. Diabetologia. 43:957-973.

2. Feldman, E.L., Stevens, M.J., and Greene, D.A 1997. Pathogenesis of diabetic neuropathy. Clin. Neurosci. 4:365-370.

3. Pham, H.T., et al. 2000. Screening techniques to identify the at risk patients for developing diabetic foot ulcers in a prospective multicentre trial. Diabetes Care. 23:606-611.

4. Fagerberg, S.E. 1959. Diabetic neuropathy, a clini$\mathrm{cal}$ and histological study on the significance of vascular affection. Acta Med. Scand. Suppl. 345:1-97.

5. Tesfaye, S., Malik, R.A., and Ward, J.D. 1994. Vascular factors in diabetic neuropathy. Diabetologia. 37:847-854.

6. Theriault, M., Dort, J., Sutherland, G., and Zochodne, D.W. 1997. Local human sural nerve blood flow in diabetic and other polyneuropathies. Brain. 120:1131-1138.

7. Apfel, S.C., et al. 2000. Efficacy and safety of recombinant human nerve growth factor in patients with diabetic polyneuropathy: a randomized controlled trial. rhNGF Clinical Investigator Group. JAMA. 284:2215-2221.
8. Sima, A.A.F., Bouchier, M., and Christensen, H. 1983. Axonal atrophy in sensory nerves of the diabetic BBWistar rat, a possible early correlate of human diabetic neuropathy. Ann. Neurol. 13:264-272.

9. Cameron, N.E., Cotter, M.A., and Low, P.A. 1991. Nerve blood flow in early experimental diabetes in rats: relation to conduction deficits. Am. J. Physiol. 261:E1-E8.

10. Stevens, E.J., Carrington, A.L., and Tomlinson, D.R. 1994. Nerve ischaemia in diabetic rats: timecourse of development, effect of insulin treatment plus comparison of streptozotocin and BB models. Diabetologia. 37:43-48.

11. Vincent, K.A., et al. 2000. Angiogenesis is induced in a rabbit model of hindlimb ischemia by naked DNA encoding an HIF-1alpha/VP16 hybrid transcription factor. Circulation. 102:2255-2261.

12. Shweiki, D., Itin, A., Soffer, D., and Keshet, E. 1992. Vascular endothelial growth factor induced by hypoxia may mediate hypoxia-initiated angiogenesis. Nature. 359:843-845.

13. Isner,J.M. 2000. Tissue responses to ischemia: local and remote responses for preserving perfusion of ischemic muscle. J. Clin. Invest. 106:615-619.

14. Aiello, L.P., et al. 1994. Vascular endothelia growth factor in ocular fluid of patients with diabetic retinopathy and other retinal disorders. $N$. Engl. J. Med. 331:1480-1487.

15. Duh, E., and Aiello, L.P. 1999. Vascular endothelial growth factor and diabetes: the agonist versus antagonist paradox. Diabetes. 48:1899-1906.

16. Xia, P., et al. 1996. Characterization of vascular endothelial growth factor's effect on the activation of protein kinase $\mathrm{C}$, its isoforms, and endothelial cell growth. J. Clin. Invest. 98:2018-2026.

17. Samii, A., Unger, J., and Lange, W. 1999. Vascular endothelial growth factor expression in peripheral nerves and dorsal root ganglia in diabetic neuropathy in rats. Neurosci. Lett. 262:159-162.

18. Schratzberger, P., et al. 2000. Favorable effect of VEGF gene transfer on ischemic peripheral neuropathy. Nat. Med. 6:405-413.

19. Schratzberger, P., et al. 2001. Reversal of experimental diabetic neuropathy by VEGF gene transfer. J. Clin. Invest. 107:1083-1092.

20. Cameron, N.E., and Cotter, M.A. 1997. Metabolic and vascular factors in the pathogenesis of diabetic neuropathy. Diabetes. 46(Suppl.):S31-S37.

21. Nakamura, J., et al. 1999. A protein kinase C-betaselective inhibitor ameliorates neural dysfunction in streptozotocin-induced diabetic rats. Diabetes. 48:2090-2095. 